\title{
ATOMIC PHYSICS
}

\section{DIELECTRONIC RECOMBINATION IN $\mathrm{He}^{+}$IONS}

\author{
J. A. Tanis, J. L. Forest and R. R. Haar \\ Western Michigan University, Kalamazoo, Michigan 49008
}

M. W. Clark and D. Schneider

Lawrence Livermore Laboratory, Livermore, California 94550

P. F. Dittner

Oak Ridge National Laboratory, Oak Ridge, Tennessee 37831

T. Ellison, C. C. Foster, W. W. Jacobs and T. Rinckel

Indiana University Cyclotron Facility, Bloomington, Indiana 47408

W. G. Graham

Queen's University, Belfast BT7 1NN, United Kingdom

J. R. Mowat

North Carolina State University, Raleigh, North Carolina 27695

M. P. Stockli

Kansas State University, Manhattan, Kansas 66506

Dielectronic recombination (DR) was investigated for $1 \mathrm{~s} \rightarrow \mathrm{nln}$ ' 1 ' transitions in $\mathrm{He}^{+}+$e-collisions. ${ }^{1} \mathrm{DR}$ occurs in an electron-ion collision when capture of the electron is accompanied by simultaneous ionic excitation, followed by subsequent radiative stabilization. The DR process is mediated by the electron-electron interaction, and proceeds through the inverse of an Auger transition; thus it is resonant for relative ion-electron velocities which correspond to exiting Auger-electron energies.

The ion storage ring and electron Cooler at IUCF were used in a "single pass" mode, i.e., the ions circled the ring, passed through the electron cooling region, were deflected by a ring magnet, and then collected in a Faraday cup. In the present case a beam of $44 \mathrm{MeV}^{4} \mathrm{He}^{+}$ions (current $\sim 100 \mathrm{nA}$ ) was merged with the electron cooling beam (current $\sim 0.3 \mathrm{~A}$ ) over an interaction length of $\sim 2.8 \mathrm{~m}$. Events resulting in DR were detected by observing neutral He atoms produced in the electron Cooler. These atoms, which exited through a $0^{\circ}$ port at the first dipole magnet following the cooler region, were observed with solid-state detectors in an $\mathrm{E}, \Delta \mathrm{E}$ arrangement, thereby allowing particle identification to separate the He atoms from background events.

For $\mathrm{He}^{+}(1 \mathrm{~s})$ ions $\mathrm{DR}$ is expected to occur for relative energies of $33-39 \mathrm{eV}$ between the ion and electron. By ramping the relative electron energy from $\left|\mathrm{E}_{r e l}\right|=0-50 \mathrm{eV}, \mathrm{DR}$ 
could be investigated for electron velocities less and greater than the ion beam velocity. For $\mathrm{E}_{r e l}=0$, a peak due to radiative recombination (RR) (inverse photoelectric effect) is expected.

In previous work ${ }^{2}$ at IUCF, a hardware problem resulted in a large energy shift in the positions of the $\mathrm{DR}$ and $\mathrm{RR}$ maxima as well as poor electron energy resolution $(\sim 20$ $\mathrm{eV}$ in the ion rest frame), thereby preventing a determination of which $\mathrm{n}$ states are dominant in the DR process. In the present measurements, the absolute electron energy was determined accurately and the resolution was improved considerably $(\sim 1.5 \mathrm{eV}$ in the ion frame). A typical spectrum is shown in Fig. 1, in which DR maxima are observed for electron velocities smaller $\left(\mathrm{v}_{e}<\mathrm{v}_{i}\right)$ and greater $\left(\mathrm{v}_{e}>\mathrm{v}_{i}\right)$ than the ion velocity and the RR maximum for $\mathrm{E}_{r e l}=0$ is also seen. The positions and widths of the DR maxima indicate that most of the dielectronic recombination occurs into states with $\mathrm{n} \geq 3$ in agreement with theoretical predictions.

This work was supported in part by the U. S. Department of Energy, Office of Basic Energy Sciences, Division of Chemical Sciences, the National Science Foundation, and the Science and Engineering Research Council (Great Britain).

1. J. A. Tanis, et al., Nucl. Instrum. Meth. Phys. Res. B43, 290 (1989).

2. J. A. Tanis, et al., Nucl. Instrum. Meth. Phys. Res. (1991), in press.

3. M. S. Pindzola, N. R. Badnell, and D. C. Griffin, Phys. Rev. A42, 282 (1990).

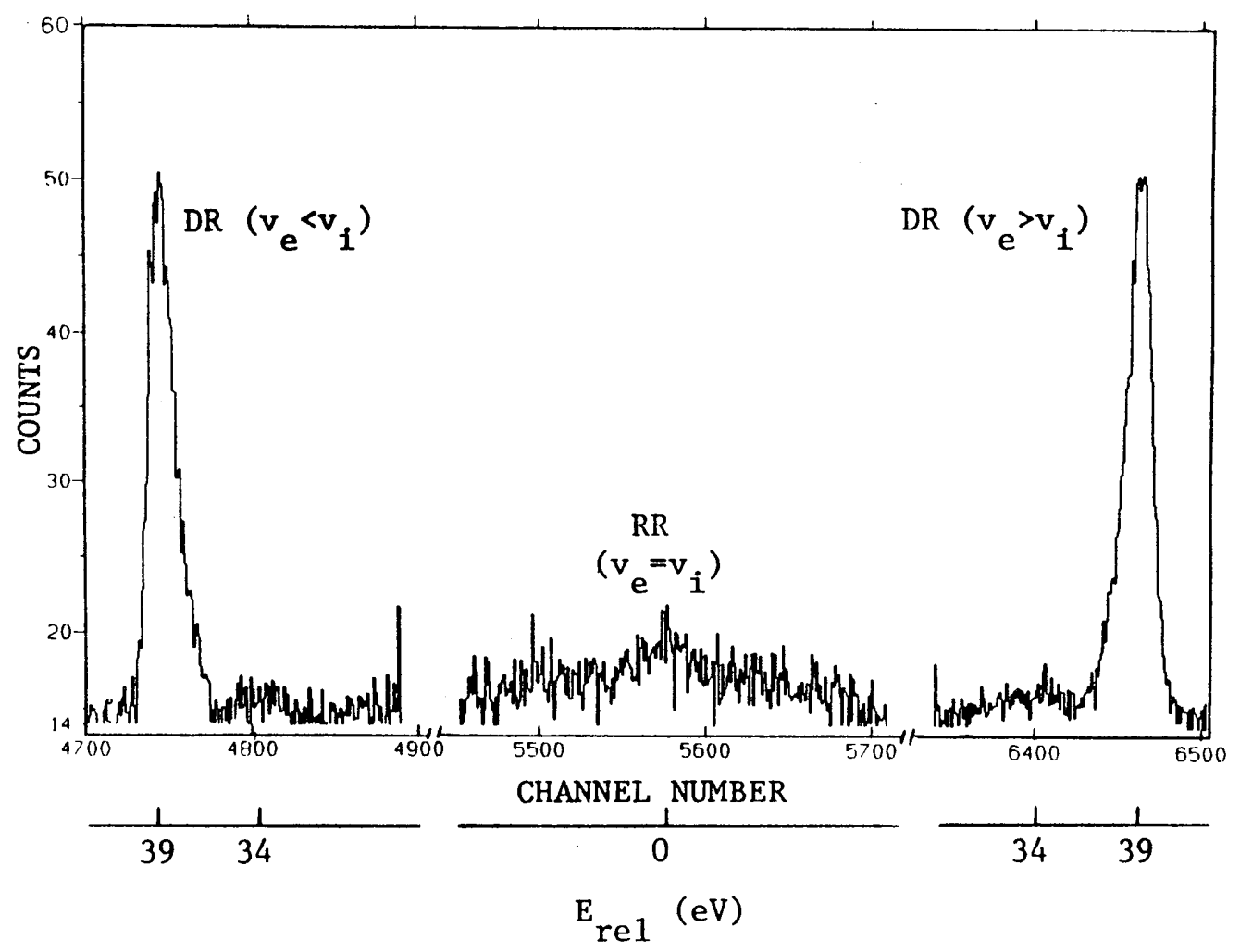

Figure 1. Intensity of neutral He atoms formed in the Cooler region as a function of electron energy. 